\title{
Upper Bounds on Inefficiency of Taxed Stackelberg Network with Cross- Nested Logit Assignment
}

\author{
Minghua Zeng ${ }^{\mathrm{a}, \mathrm{b}, *}$ and Yinsong Wang ${ }^{\mathrm{b}}$ \\ ${ }^{a}$ School of Railway Tracks and Transportation, East China Jiao Tong University, Nanchang 330013, P.R. China; \\ ${ }^{b}$ School of transportation Engineering, Tongji University, Shanghai 201804, P.R. China
}

\begin{abstract}
Upper bound of efficiency loss is a valuable issue for transport network design and planning. This paper initially explores it in a taxed stochastic traffic network whose equilibrium flow pattern is deduced by a cross-nested Logit (CNL) flow assignment model, and a centrally controlling Stackelberg strategy. With the assumptions of separability, nondecreasingness, and convexity of the link time function and the fixed origin-destination (OD) demand of network, the equivalent variational inequality for a CNL-based stochastic user equilibrium (CNL-SUE) model is established and first used to obtain upper bounds on Stackelberg network inefficiency. Further, for low-degree link time function such as Bureau of Public Roads and the affine forms, their inefficiency upper bounds are analyzed with some meaningful results.
\end{abstract}

Keywords: Cross-nested logit, price of anarchy, stackelberg, stochastic user equilibrium, tax scheme, transportation.

\section{INTRODUCTION}

How is the efficiency loss of users' selfish route choice behaviors affected by traffic assignment model, stochastic factor in traveler route selection, routing strategies, and tax schemes? Attempting to contribute to this practical end, this paper makes great efforts to investigate their combined impacts on the inefficiency upper bound.

The price of anarchy concept dates back to 1999 when Koutsoupias and Papadimitrious [1] first proposed to determine the efficiency losses caused by the non-cooperative behavior of users in telecommunication network. The POA coined by Papadimitriou (2001) [2] for a non-atomic congestion game is determined by looking for the worst possible ratio between the total cost incurred by players in an user equilibrium (UE) situation and of a system optimum (SO). Hereafter the efficiency loss and its upper bound of traffic equilibrium received much attention [3-5]. Roughgarden and Tardos (2002) [3] first used the concept of POA [1] to upper bound the efficiency loss and proved that the total travel time of a UE is at most $1 / 3$ higher than in a SO if the latency functions are linear with nonnegative coefficients. Chau and Sim (2003) [6] and Yang et al. (2010) [5] studied the efficiency loss of transportation equilibrium problem with elastic demand.

None of the above researches considers Stackelberg routing strategies. Among Stackelberg strategies, the SCALE is a popular one whose effectiveness has been analyzed [7, 8]. Scholars have investigated the impact of Stackelberg routing to reduce the UE cost in network routing $[7,9,10]$. In simplest graphs with parallel links and latency functions of special simplified form as the inverse of the minus of link capacity and link flow, Korilis et al. (1997) [9] first applied Stackelberg strategy controlling a fraction of flow as a means to improve system performance considering atomic, unsplittable routing decision. From then on, some works of Stackelberg policy developed to improve the POA such as Roughgarden (2004) [11], Stier-Moses (2004) [12], Karakostas and Kolliopoulos (2009) [10] on parallel-link or general graph, primarily for linear latency functions.

Furthermore, due to an absence of road pricing that originated from Pigou (1920) [13], the demand-supply equilibrium of road network settles at a suboptimal point bringing about the negative externality. Such inefficiency pervades many road networks during peak hours. For that reason, there emerge few researches focusing on the impact of tax schemes on POA $[5,14]$. Such works involve a demand-adjusting tax-charging policy under currently deteriorated urban traffic condition to obtain different and intensive insights into the POA of transport network.

Despite of the employment of Stackelberg strategies or tax schemes, the existing researches on POA bound mainly focus on deterministic traffic assignment, i.e. UE, while the few on stochastic user equilibrium (SUE) assignment just adopting multinomial Logit (MNL) model [15], the simplest one in Logit families. For example, Guo et al. (2010) [16] gave the bound of POA of Logit-based stochastic user equilibrium, Yu et al. (2009) [17] investigated the upper bound of SUE's inefficiency. It is well known that different models lead to network different flow assignment pattern and hence different inefficiency. Previous studies on price of anarchy (POA) usually adopt. However, UE is so simple, and MNL cannot deal with path overlapping characteristics, which could be perfectly. Therefore, it is of great significance to employ cross-nested Logit (CNL) model [18, 19] that can capture path correlation to find POA upper bound in network 
flow assignment. CNL model has been mentioned for the first time by Vovsha (1997) [20] in the context of a mode choice survey in Israel, and be applied to route choice problem by Prashker and Bekhor (1998) [18], and so on.

Since most researches on POA bound apply MNL model, presented in the absence of either Stackelberg strategy, or stochastic user equilibrium, or tax scheme, CNL-SUE induced by Stackelberg strategy in this paper cooperates with weak Stackelberg policy and tax scheme to improve the upper bound of inefficiency stemming from the stochastic selfish route selection behavior. Network tolls imposed by a central authority on edges help to drive the Stackelberginduced stochastic user equilibrium towards system performance improving state. Section 2 presents the problem studied with basic symbols and concepts. Assuming that link time function is separable and monotonic and that OD demand is fixed, Section 3 formulates the equivalent variational inequalities (VI) for the CNL-based SUE model induced by weak Stackelberg strategy and tax scheme. Further, Section 4 analyzes and establishes POA upper bound on taxed Stackelberg CNL-SUE. In Section 5, POA upper bounds are analyzed for the cases of low-degree link time functions, i.e. typical BPR function and its affine form. Section 6 gives some concluding remarks.

\section{STACKELBERG STOCHASTIC EQUILIBRIUM IN TAXED NETWORK}

Let $\boldsymbol{G}=(\boldsymbol{V}, \boldsymbol{E})$ denote a traffic network, where $\boldsymbol{V}$ and $\boldsymbol{E}$ are the set of vertices and edges respectively. Let $\boldsymbol{W}=\{w\}$ be a set of OD pairs, $\boldsymbol{R}_{w}$ the set of all simple paths of $w \in \boldsymbol{W}, q^{w}>0$ the travel demand between $w$ with $\boldsymbol{q}=\left(q^{w}\right) \in \stackrel{+}{|w| \times 1}_{+}$as demand vector, where + represents the set of nonnegative real number. Let $f_{e r}^{w}$ be the traffic volume on path $r \in \boldsymbol{R}_{w}$ passing link $e \in \boldsymbol{E}_{r}$, with path flow

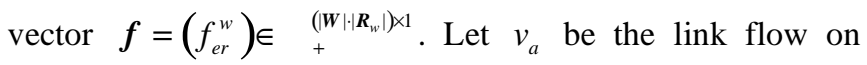
edge $a \in \boldsymbol{E}$, and its corresponding vector $\boldsymbol{v}=\left(v_{a}\right) \in \underset{+}{|\boldsymbol{E}| \times 1}$.

Let $u=\left(u_{a}: a \in \boldsymbol{E}\right) \in \underset{+}{|\boldsymbol{E}|}$ be a general tax scheme, where $u_{a}$ is the tax charge on link $a \in \boldsymbol{E}$ in equivalent time unit. It has been recognized that the second-best pricing scheme is more technically and practically feasible. In reality, tax charge for a link can be either less or greater than the corresponding congestion externality in a second-best pricing scheme, so assume $u_{a}=k_{a}\left(\alpha v_{a}^{\text {so }}+v_{a}^{*}\right) t_{a}^{\prime}\left(\alpha v_{a}^{\text {so }}+v_{a}^{*}\right)$, where $k_{a} \in[0,2]$ is a tax coefficient. Let $t_{a}=t_{a}\left(v_{a}\right) \in \mathcal{T}$ denote a differentiable increasing convex function of flow $v_{a}$ (separable case), i.e. $d t_{a}\left(v_{a}\right) / d v_{a} \geq 0$ and $d^{2} t_{a}\left(v_{a}\right) / d v_{a}^{2} \geq 0$, $a \in E$, with a natural property that $v t(v)$ is convex in $v$, where $\mathcal{T}$ is a given class of link time functions, $\mathcal{T}$ comprises a class of BPR functions and simple affine functions. Then link cost function $c_{a}\left(v_{a}\right)$ on link $a \in \boldsymbol{E}$ can be expressed as $c_{a}\left(v_{a}\right)=t_{a}\left(v_{a}\right)+u_{a}$.
UE is inefficient achieved from noncooperative selfish behaviors, without consideration of entire social objectives, which leads to network performance degradation. As to SUE assignment where each user also cannot decrease his travel cost by unilaterally deviating from his path, it is the expansion of UE assignment. A Stackelberg stochastic user equilibrium problem, for traffic network, is defined by embedding SUE traffic model into a Stackelberg network framework, with leader system pursuing optimum and follower system stochastic Nash equilibrium. Leading users of a fixed fraction $\alpha$ centrally controlled by a network manager choose optimal paths in any desired way to minimize total system travel time, and the others acting as followers influenced by the leaders attempt to choose their routes under SUE assignment. A weak Stackelberg strategy [21] is defined as a feasible path flow pattern $\bar{f}$ that satisfies $\sum_{r \in \boldsymbol{R}_{w}} \bar{f}_{e r}^{w}=\alpha q^{w}, \forall w \in \boldsymbol{W}$. An opt-restricted Stackelberg strategy $\overline{\boldsymbol{f}}$ is such a strategy that meets $\bar{v}_{a} \leq v_{a}^{\mathrm{so}}, \forall a \in \boldsymbol{E}$, the use of which has been proved not to increase the price of anarchy [22]. According to the proof by Smith (1979) [23], a lemma is given as following.

Lemma 1. With a Stackelberg strategy $\overline{\boldsymbol{f}}$, the Nash flow $\boldsymbol{f}^{*}$ induced by $\overline{\boldsymbol{f}}$ in conjunction with taxes $u$, can be characterized by the following variational inequality: $f^{*}$ is a Nash flow induced by $\bar{f}$ if and only if for arbitrary $\boldsymbol{x}$ satisfying demands $(1-\alpha) q^{w}, w \in \boldsymbol{W}$ such that

$$
\begin{aligned}
\sum_{a \in \boldsymbol{E}} c_{a}\left(\bar{v}_{a}+v_{a}^{*}\right)\left(v_{a}^{*}-x_{a}\right) & \leq 0 \\
\text { Let } \quad T\left(\overline{\boldsymbol{v}}+\boldsymbol{v}^{*}\right) & =\sum_{a \in \boldsymbol{E}} t_{a}\left(\bar{v}_{a}(u)+v_{a}^{*}(u)\right)\left(\bar{v}_{a}(u)+v_{a}^{*}(u)\right)
\end{aligned}
$$

denote the total system travel time at Stackelberg Nash equilibrium assignment. We are interested in the price of anarchy defined by $\rho^{\text {eq }}=T\left(\overline{\boldsymbol{v}}+\boldsymbol{v}^{*}\right) / T^{\text {so }}$, where $T^{\text {so }}=T\left(\boldsymbol{v}^{\text {so }}\right)$ denotes the optimal total system travel time, and $\boldsymbol{v}^{\text {so }}=\arg \min _{\boldsymbol{v} \in \check{U}_{v}} \sum_{a \in E} t_{a}\left(v_{a}\right) v_{a}$ that is unique due to the strict convexity of $t_{a}\left(v_{a}\right) v_{a}$.

Stochastic user equilibrium models are established based on the assumption of variations in travelers' perception, due to perhaps different cognition of the traffic network, of the travel costs along the link. The perceived travel costs can be expressed as the extension of travel cost functions in deterministic traffic model by including random parts. The most widely used SUE model is the multinomial Logit (MNL) model obtained assuming that the random terms are independently and identically distributed Gumbel variables. Other than MNL, Cross-nested Logit (CNL) model is another typical case in Logit family. MNL is simple but cannot capture path correlation structure, while CNL is just the opposite. CNL [18, 19] models correlation through discrete choice model and the distribution assumption of error items. Prashker and Bekhor (1998) [18] applied CNL to route choice problem. CNL model allow more flexible error structure by applying a two-level tree structure. We put the CNL 
choice probability of route $r$ between OD pair $w$ in hierarchical road network as follows

$$
P_{r}^{w}=\frac{\exp \left[-\theta c_{r}^{w}+\ln \sum_{e \in E_{r}} \alpha_{e r}^{w}\left(\sum_{j} \alpha_{e j}^{w} \exp \left(-\theta c_{j}^{w}\right)\right)^{\mu-1}\right]}{\sum_{l \in \boldsymbol{R}_{w}} \exp \left[-\theta c_{l}^{w}+\ln \sum_{e \in E_{r}} \alpha_{e l}^{w}\left(\sum_{j} \alpha_{e j}^{w} \exp \left(-\theta c_{j}^{w}\right)\right)^{\mu-1}\right]}
$$

where $\theta$ is a positive scaling factor related to the variances in the perceived travel costs, larger values implying less between-traveler perception variation; $\boldsymbol{E}_{r}$ is the set of links constituting route $r ; \alpha_{e r}^{w}$ denotes allocating parameter to describe how likely alternative route $r$ is allocated to the nest $e$ that belongs to the route, and satisfies $\alpha_{e r}^{w} \geq 0$, $(\forall r) \sum_{e} \alpha_{e r}^{w}=1 . \mu_{e} \in(0,1]$ is dissimilarity parameter of nest $e$, representing the similarity or correlation among stochastic items of alternative routes in the nest; $\mu$ indicates the nested degree; allocating parameters are calculated by network topologies [24], defined as (3) by link and path "length".

$\alpha_{e r}^{w}=\left(L_{a} / L_{r}\right)^{\gamma_{1}} \delta_{e r}^{w}, \forall e \in \boldsymbol{E}_{r}, r \in \boldsymbol{R}_{w}$

where $\gamma_{1}$ is a parameter to be calibrated reflecting travelers' perceived similarity among paths, and $\delta_{a r}^{w}$ is the element of

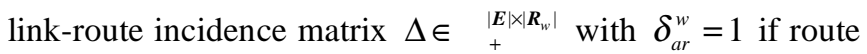
$r$ uses link $a$ and zero otherwise.

\section{VI FOR CNL-SUE WITH STACKELBERG STRAT- EGY AND TAX SCHEME}

In terms of the assumption that the link time function is separable and monotonically increasing with link flow, and that the network OD demand is fixed, the equivalent variational inequality formulation for the cross-nested Logitbased SUE model is given as following.

Theorem 1. The stochastic user equilibrium flow $f^{*}$ induced by $\bar{f}$ with the use of CNL-SUE model can to be characterized by the following variational inequality: $f^{*} \in \boldsymbol{\Omega}_{f}$ is a stochastic user equilibrium path flow pattern induced by $\bar{f}$ if and only if for all flows $f$ satisfying demands $(1-\alpha) q^{w}, w \in \boldsymbol{W}$ such that

$$
\begin{aligned}
& \sum_{w \in W} \sum_{r \in \boldsymbol{R}_{w}} c_{r}^{w}\left(\bar{f}_{e r}^{w}, f_{e r}^{w^{*}}\right)\left(f_{e r}^{w^{*}}-f_{e r}^{w}\right)+\sum_{w \in W} \sum_{r \in \boldsymbol{R}_{w}}\left(\frac{1-\mu}{\theta} \ln \left((1-\alpha) q^{w}\right)+\right. \\
& \left.+\frac{\mu}{\theta} \ln f_{e r}^{w^{*}}-\frac{1}{\theta} \ln \alpha_{e r}^{w}\right)\left(f_{e r}^{w^{*}}-f_{e r}^{w}\right) \leq 0, \boldsymbol{f} \in \boldsymbol{\Omega}_{f}
\end{aligned}
$$

where $c_{r}^{w}=\sum_{a \in E} c_{a}\left(v_{a}\right) \delta_{a r}^{w}=\sum_{a \in E}\left(t_{a}\left(v_{a}\right)+u_{a}\right) \delta_{a r}^{w}$; and

$$
\boldsymbol{\Omega}_{f}=\left\{f_{e r}^{w} \mid \sum_{e \in E_{r}} \sum_{r \in \boldsymbol{R}_{w}} f_{e r}^{w}=q_{w}, f_{e r}^{w} \geq 0, \forall e \in \boldsymbol{E}_{r}, r \in \boldsymbol{R}_{w}, w \in \boldsymbol{W}\right\}
$$

is the nonempty, convex, and compact set of feasible path flow pattern.
Proof. According to Prashker and Bekhor (1998) [18], the above cross-nested Logit-based SUE model can be formulated as the following equivalent mathematical programming problem (MP1)

(MP1)

$\min \mathrm{Z}_{1}(\boldsymbol{f})=\sum_{a \in E} \int_{0}^{v_{a}} c_{a}(v) d v+\frac{\mu}{\theta} \sum_{w \in W} \sum_{e \in E_{r}} \sum_{r \in \boldsymbol{R}_{w}} f_{e r}^{w} \ln \frac{f_{e r}^{w}}{\left(\alpha_{e r}^{w}\right)^{1 / \mu}}+$

$$
\begin{array}{ll}
\text { s.t. } & \sum_{e \in \boldsymbol{E}_{r}} \sum_{r \in \boldsymbol{R}_{w}} f_{e r}^{w}=q^{w}, \forall w \\
& v_{a}=\sum_{w \in W} \sum_{r \in \boldsymbol{R}_{w}} \sum_{e \in \boldsymbol{E}_{r}} f_{e r}^{w} \delta_{r a}^{k}, \forall a
\end{array}
$$$$
f_{e r}^{w} \geq 0, \forall e, r, w
$$

$$
\frac{1-\mu}{\theta} \sum_{w \in W} \sum_{e \in E_{r}}\left(\sum_{r \in \boldsymbol{R}_{w}} f_{e r}^{w}\right) \ln \left(\sum_{r \in \boldsymbol{R}_{w}} f_{e r}^{w}\right)
$$

Let $\pi^{w}$ be the Lagrange multiplier (dual variable) of constraint $\sum_{e \in \boldsymbol{E}_{r}} \sum_{r \in \boldsymbol{R}_{w}} f_{e r}^{w}=q_{w} . \pi^{w}$ represents the expected minimum conceptual travel cost. The Lagrange function of MP-CNL is formulated as

$$
L(\boldsymbol{f}, \boldsymbol{\pi})=\mathrm{Z}(\boldsymbol{f})+\sum_{w \in W} \pi^{w}\left(q^{w}-\sum_{e \in \boldsymbol{E}_{r}} \sum_{r \in \boldsymbol{R}_{w}} f_{e r}^{w}\right)
$$

In terms of the first-order optimality conditions, we have

$f_{e r}^{w} \frac{\partial L}{\partial f_{e r}^{w}}=0, \frac{\partial L}{\partial f_{e r}^{w}} \geq 0, f_{e r}^{w} \geq 0 ; q^{w} \frac{\partial L}{\partial q^{w}}=0, \frac{\partial L}{\partial q^{w}} \geq 0, q^{w} \geq 0 ; \frac{\partial L}{\partial \pi^{w}}=0, \pi^{w} \geq 0$

So for fixed traffic demand, this conditions generates

$\frac{\partial L}{\partial f_{e r}^{w}}=c_{r}^{w}+\frac{\mu}{\theta} \ln \frac{f_{e r}^{w}}{\left(\alpha_{e r}^{w}\right)^{1 / \mu}}+\frac{1-\mu}{\theta} \ln \left(\sum_{r \in R_{w}} f_{e r}^{w}\right)+\frac{1}{\theta}-\pi^{w}$

Furthermore, we get

$$
\begin{aligned}
& \sum_{w \in W} \sum_{r \in R_{w}}\left(c_{r}^{w}\left(f^{*}\right)+\frac{1-\mu}{\theta} \ln \left(\sum_{r \in R_{w}} f_{e r}^{w^{*}}\right)+\frac{\mu}{\theta} \ln \frac{f_{e r}^{w^{*}}}{\left(\alpha_{e r}^{w}\right)^{1 / \mu}}\right)\left(f_{e r}^{w}-f_{e r}^{w^{*}}\right) \geq 0, \\
& \forall \boldsymbol{f} \in I_{s} f \\
& \quad \text { and }
\end{aligned}
$$

$f_{e r}^{w}\left(\sum_{r \in \boldsymbol{R}_{w}} f_{e r}^{w}\right)^{\frac{1}{\mu}-1}=\left(\alpha_{e r}^{w}\right)^{1 / \mu}\left(\exp \left(\theta \pi^{w}-\theta c_{r}^{w}-1\right)\right)^{1 / \mu}$

and then taking summation of (10) over all path of $\boldsymbol{R}_{w}$, we reformulate to get

$$
\left(\sum_{r \in \boldsymbol{R}_{w}} f_{e r}^{w}\right)^{1 / \mu}=\sum_{r \in \boldsymbol{R}_{w}}\left(\alpha_{e r}^{w}\right)^{1 / \mu}\left(\exp \left(\theta \pi^{w}-\theta c_{r}^{w}-1\right)\right)^{1 / \mu}
$$

So, under the condition of selecting link (nest) $e \in \boldsymbol{E}_{r}$, conditional choice probability of route $r \in \boldsymbol{R}_{w}$ can be obtained by (10) and (11) as the follows

$$
P(r \mid e)=\frac{f_{e r}^{w}}{\sum_{r \in \boldsymbol{R}_{w}} f_{e r}^{w}}=\frac{\left(\alpha_{e r}^{w} \exp \left(-\theta c_{r}^{w}\right)\right)^{1 / \mu}}{\sum_{r \in \boldsymbol{R}_{w}}\left(\alpha_{e r}^{w} \exp \left(-\theta c_{r}^{w}\right)\right)^{1 / \mu}}
$$


where $\sum_{r \in \boldsymbol{R}_{w}} f_{e r}^{w}$ is the traffic volume on link $e \in \boldsymbol{E}_{r}$ of route $r \in \boldsymbol{R}_{w}$ between OD pair $w \in \boldsymbol{W}$.

Taking a $\mu$ power for (11), we have

$$
\sum_{r \in \boldsymbol{R}_{w}} f_{e r}^{w}=\left[\sum_{r \in \boldsymbol{R}_{w}}\left(\alpha_{e r}^{w}\right)^{1 / \mu}\left(\exp \left(\theta \pi^{w}-\theta c_{r}^{w}-1\right)\right)^{1 / \mu}\right]^{\mu}
$$

Taking summation over the set of $\boldsymbol{E}_{r}$ for (13) results in

$$
\sum_{e \in \boldsymbol{E}_{r}} \sum_{r \in \boldsymbol{R}_{w}} f_{e r}^{w}=\sum_{e \in \boldsymbol{E}_{r}}\left[\sum_{r \in \boldsymbol{R}_{w}}\left(\alpha_{e r}^{w}\right)^{1 / \mu}\left(\exp \left(\theta \pi^{w}-\theta c_{r}^{w}-1\right)\right)^{1 / \mu}\right]^{\mu}
$$

Then, choice probability of link (nest) $e \in \boldsymbol{E}_{r}$ can be obtained by (13) and (14) as the follows

$$
P(e)=\frac{\sum_{r \in \boldsymbol{R}_{w}} f_{e r}^{w}}{\sum_{e \in \boldsymbol{E}_{r}} \sum_{r \in \boldsymbol{R}_{w}} f_{e r}^{w}}=\frac{\left[\sum_{r \in \boldsymbol{R}_{w}}\left(\alpha_{e r}^{w} \exp \left(-\theta c_{r}^{w}\right)\right)^{1 / \mu}\right]^{\mu}}{\sum_{e \in \boldsymbol{E}_{r}}\left[\sum_{r \in \boldsymbol{R}_{w}}\left(\alpha_{e r}^{w} \exp \left(-\theta c_{r}^{w}\right)\right)^{1 / \mu}\right]^{\mu}}
$$

Therefore, route choice probability for $r \in \boldsymbol{R}_{w}$, i.e. $P_{r}^{w}$ can be obtained by $\sum_{e \in \boldsymbol{E}_{r}} P(e) P(r \mid e)$.

In view of the assignment of route flow $f_{e r}^{w^{*}}=(1-\alpha) q^{w} P_{r}^{w}, \quad$ we $\sum_{r \in \boldsymbol{R}_{w}} f_{e r}^{w^{*}}=\sum_{r \in \boldsymbol{R}_{w}}(1-\alpha) q^{w} P_{r}^{w}$, i.e.,

$\ln \left(\sum_{r \in \boldsymbol{R}_{w}} f_{e r}^{w^{*}}\right)=\ln \left((1-\alpha) q^{w}\right)+\ln \sum_{r \in \boldsymbol{R}_{w}} P_{r}^{w}=\ln \left((1-\alpha) q^{w}\right)$

Thus, according to lemma 1, substituting (16) into (9), we get that $f^{*}$ is a stochastic user equilibrium flow induced by a Stackelberg strategy $\bar{f}$ if and only if for all flows $\boldsymbol{f}$ satisfying demands $(1-\alpha) q^{w}, w \in \boldsymbol{W}$ such that

$$
\begin{aligned}
& \sum_{w \in \boldsymbol{W}} \sum_{r \in \boldsymbol{R}_{w}} c_{r}^{w}\left(\bar{f}_{e r}^{w}, f_{e r}^{w^{*}}\right)\left(f_{e r}^{w^{*}}-f_{e r}^{w}\right)+\sum_{w \in \boldsymbol{W}} \sum_{r \in \boldsymbol{R}_{w}}\left(\frac{1-\mu}{\theta} \ln \left((1-\alpha) q^{w}\right)+\right. \\
& \left.\frac{\mu}{\theta} \ln f_{e r}^{w^{*}}-\frac{1}{\theta} \ln \alpha_{e r}^{w}\right)\left(f_{e r}^{w^{*}}-f_{e r}^{w}\right) \leq 0, \boldsymbol{f} \in \boldsymbol{\Omega}_{f}
\end{aligned}
$$

i.e., variational inequality (4) follows. So the proof is completed.

\section{GENERAL RESULTS OF POA UPPER BOUND ON TAXED STACKELBERG CNL-SUE}

Applying the above variational inequality (17) and the following definition, we analyze the upper bound on Stackelberg network inefficiency under CNL-SUE and tax scheme.

For any link time function $t_{a}=t_{a}\left(v_{a}\right)$ and Stackelberg link flow $\bar{v}_{a}$, we define nonnegative value as

$$
\gamma\left(t_{a} ; \bar{v}_{a}, u_{a}, \lambda\right)=\sup _{\substack{v_{a}^{\mathrm{s}}, v_{a}^{*} \geq 0 \\ t_{a}\left(\bar{v}_{a}+v_{a}^{*}\right)\left(\bar{v}_{a}+v_{a}^{*}\right)}} \frac{\left(t_{a}\left(\bar{v}_{a}+v_{a}^{*}\right)-\lambda t_{a}\left(v_{a}^{\mathrm{so}}\right)\right) v_{a}^{\mathrm{so}}-\left(\bar{v}_{a}+v_{a}^{*}-u_{a}^{\mathrm{so}}\right.}{t_{a}}
$$

where $0 / 0=0$ by convention, $\lambda \in[0,1]$. For a given optrestricted strategy $\overline{\boldsymbol{v}}$, a class $\mathcal{T}$ of link time functions and a tax scheme $u$, we further define

$$
\gamma(\mathcal{T}, \bar{v}, u, \lambda)=\max _{t_{a} \in \mathcal{T}, a \in E} \gamma\left(t_{a} ; \bar{v}_{a}, u_{a}, \lambda\right)
$$

Theorem 2. The cost of induced network flow by the combination of the cost of system optimum and the induced link flows is bounded with coefficients $\lambda$ and $\gamma$ as following

$$
\begin{aligned}
T\left(\overline{\boldsymbol{v}}+\boldsymbol{v}^{*}\right) & \leq \sum_{a \in E} \lambda t_{a}\left(v_{a}^{\mathrm{so}}\right) v_{a}^{\mathrm{so}}+\sum_{a \in E} \gamma\left(t_{a} ; \bar{v}_{a}, u_{a}, \lambda\right) t_{a}\left(\bar{v}_{a}+v_{a}^{*}\right)\left(\bar{v}_{a}+v_{a}^{*}\right)+F\left(\boldsymbol{f}, \boldsymbol{f}^{*}\right) \\
& =\lambda T^{\mathrm{so}}+\gamma(\mathcal{T}, \overline{\boldsymbol{v}}, u, \lambda) T\left(\overline{\boldsymbol{v}}+\boldsymbol{v}^{*}\right)+F\left(\boldsymbol{f}, \boldsymbol{f}^{*}\right)
\end{aligned}
$$

Proof. Let $\boldsymbol{f}=\boldsymbol{f}^{\text {so }}-\overline{\boldsymbol{f}}$ be the flow that remains in if $\overline{\boldsymbol{f}}$ is removed from the optimal flow $\boldsymbol{f}^{\text {so }}, \boldsymbol{f}$ is a flow satisfying demands $(1-\alpha) q^{w}, \quad \forall w \in W$. Setting $\boldsymbol{f}=\boldsymbol{f}^{\text {so }}-\overline{\boldsymbol{f}}$ $\left(\boldsymbol{v}=\boldsymbol{v}^{\text {so }}-\overline{\boldsymbol{v}}\right)$ in formula (17) generates

$T\left(\overline{\boldsymbol{v}}+\boldsymbol{v}^{*}\right)-\sum_{a \in \boldsymbol{E}} t_{a}\left(\bar{v}_{a}+v_{a}^{*}\right) v_{a}^{\mathrm{so}}+\sum_{a \in \boldsymbol{E}}\left(v_{a}^{*}+\bar{v}_{a}-v_{a}^{\mathrm{so}}\right) u_{a}-F\left(\boldsymbol{f}, \boldsymbol{f}^{*}\right) \leq 0$.

where

$$
F\left(\boldsymbol{f}, \boldsymbol{f}^{*}\right) \quad \sum_{w \in W} \sum_{r \in \boldsymbol{R}_{w}}\left(\frac{1-\mu}{\theta} \ln \left((1-\alpha) q^{w}\right)+\frac{\mu}{\theta} \ln f_{e r}^{w^{*}}-\frac{1}{\theta} \ln \alpha_{e r}^{w}\right)
$$

$\left(f_{e r}^{w, s o}-f_{e r}^{w^{*}}-\bar{f}_{e r}^{w}\right)$.

Thus, for any opt-restricted strategy $\bar{f}$, the following inequality holds

$$
T\left(\overline{\boldsymbol{v}}+\boldsymbol{v}^{*}\right) \leq \sum_{a \in \boldsymbol{E}} v_{a}^{\mathrm{so}} t_{a}\left(\bar{v}_{a}+v_{a}^{*}\right)-\sum_{a \in \boldsymbol{E}}\left(v_{a}^{*}+\bar{v}_{a}-v_{a}^{\mathrm{so}}\right) u_{a}+F\left(\boldsymbol{f}, \boldsymbol{f}^{*}\right)
$$

Accompanying with the definition of $\gamma_{a}\left(t_{a} ; \bar{v}_{a}, u_{a}, \lambda\right)$ by (18) and variational inequality (21), the cost of induced network flow can be bounded as formula (20).

Remark1. Formula (20) can be expressed equivalently as price of anarchy

$$
\frac{T\left(\overline{\boldsymbol{v}}+\boldsymbol{v}^{*}\right)}{T^{\mathrm{so}}} \leq \frac{\lambda}{1-\gamma(\mathcal{T}, \overline{\boldsymbol{v}}, u, \lambda)}+\frac{1}{(1-\gamma(\mathcal{T}, \overline{\boldsymbol{v}}, u, \lambda)) T^{\mathrm{so}}} F\left(\boldsymbol{f}, \boldsymbol{f}^{*}\right)
$$

Assume a class of link time functions $\mathcal{T}_{p}=\left\{t(\boldsymbol{v}) \mid t(c \boldsymbol{v}) \geq c^{p} t(\boldsymbol{v}), \forall c \in[0,1]\right\}$, where $\quad p \geq 1$ is an integer, and $\mathcal{T}_{p}$ contains polynomials of degree at most $p$, $t(\boldsymbol{v})=\sum_{i=0}^{p} a_{i} v^{i}$ with nonnegative coefficients $a_{0}=t(0) \geq 0$, $a_{i}=t^{(i)}(0) \geq 0, i=1,2, \ldots, p$. At the same time, assume the application of a SCALE policy, a weak one defined by setting $\bar{v}_{a}=\alpha v_{a}^{\text {so }}, \forall a \in \boldsymbol{E}$, which is equivalent to setting $\bar{f}_{e r}^{w}=\alpha f_{e r}^{w, \text { so }}$.

For the case of link time function $t_{a}\left(v_{a}\right) \in \mathcal{T}_{p}$, we will investigate the upper bound on inefficiency of stochastic network equilibrium under the controlling of Stackelberg with tax schemes imposed on the traffic network. 
Lemma 2. If $t(v) \in \mathcal{T}_{d}$, then $\gamma\left(\mathcal{T}, \alpha v_{a}^{\mathrm{so}}, u, \lambda\right) \leq$ $\max \left\{\frac{1}{\alpha}(1-\lambda)-\left(1-\frac{1}{\alpha}\right) k_{a}, \frac{d}{d+1}\left(1+k_{a}\right)\left(\frac{1+k_{a}}{(d+1) \lambda}\right)^{1 / d}-k_{a}\right\}$.

Proof. By the definition of $\gamma\left(t_{a} ; \bar{v}_{a}, u_{a}, \lambda\right)$ in (18),

$$
\begin{gathered}
\quad \gamma\left(t_{a} ; \alpha v_{a}^{\mathrm{so}}, u_{a}, \lambda\right)= \\
: \sup _{v_{a}^{\mathrm{so}}, v_{a}^{*} \geq 0} \frac{\left(t_{a}\left(\alpha v_{a}^{\mathrm{so}}+v_{a}^{*}\right)-\lambda t_{a}\left(v_{a}^{\mathrm{so}}\right)\right) v_{a}^{\mathrm{so}}-\left(\alpha v_{a}^{\mathrm{so}}+v_{a}^{*}-v_{a}^{\mathrm{so}}\right) u_{a}}{t_{a}\left(\alpha v_{a}^{\mathrm{so}}+v_{a}^{*}\right)\left(\alpha v_{a}^{\mathrm{so}}+v_{a}^{*}\right)}
\end{gathered}
$$

analyses are carried out considering two cases, namely, (i) $\alpha v_{a}^{\mathrm{so}}+v_{a}^{*} \geq v_{a}^{\mathrm{so}}$, and (ii) $\alpha v_{a}^{\mathrm{so}}+v_{a}^{*}<v_{a}^{\mathrm{so}}$.

(i) $\alpha v_{a}^{\text {so }}+v_{a}^{*} \geq v_{a}^{\text {so }}$. Set $\varphi=v_{a}^{\text {so }} /\left(\alpha v_{a}^{\text {so }}+v_{a}^{*}\right), \varphi \in[0,1]$. Taking into account the expressions of $u_{a}$, and taking a notice of definition of $\mathcal{T}_{1}$, we have

$$
\begin{aligned}
& \gamma\left(t_{a} ; \alpha v_{a}^{\mathrm{so}}, u_{a}, \lambda\right)=\sup _{v_{a}^{\mathrm{so}}, v_{a}^{v_{a} \geq 0, \varphi \in[0,1]}} \\
& \frac{\left(t_{a}\left(\alpha v_{a}^{\mathrm{so}}+v_{a}^{*}\right)-\lambda t_{a}\left(\varphi\left(\alpha v_{a}^{\mathrm{so}}+v_{a}^{*}\right)\right)\right) \varphi-(1-\varphi) k_{a}\left(\alpha v_{a}^{\mathrm{so}}+v_{a}^{*}\right) t_{a}^{\prime}\left(\alpha v_{a}^{\mathrm{so}}+v_{a}^{*}\right)}{t_{a}\left(\alpha v_{a}^{\mathrm{so}}+v_{a}^{*}\right)} \\
& \leq \max _{v_{a}^{\mathrm{so}}, v_{a}^{*} \geq 0, \varphi \in[0,1]}\left\{\varphi\left(1-\lambda \varphi^{p}\right)-(1-\varphi) k_{a} \frac{\left(\alpha v_{a}^{\mathrm{so}}+v_{a}^{*}\right) t_{a}^{\prime}\left(\alpha v_{a}^{\mathrm{so}}+v_{a}^{*}\right)}{t_{a}\left(\alpha v_{a}^{\text {so }}+v_{a}^{*}\right)}\right\}
\end{aligned}
$$

Taking a notice of the monotonicity and convexity of link time function, and the fact that the highway sections used by so many vehicles as $\alpha v_{a}^{\text {so }}+v_{a}^{*} \geq v_{a}^{\text {so }}$ can be thought as heavily or at least lightly congested implies $\alpha v_{a}^{\text {so }}+v_{a}^{*} \geq C_{a}$, it is naturally that $t_{a}\left(\alpha v_{a}^{\mathrm{so}}+v_{a}^{*}\right) \leq\left(\alpha v_{a}^{\mathrm{so}}+v_{a}^{*}\right) t_{a}^{\prime}\left(\alpha v_{a}^{\mathrm{so}}+v_{a}^{*}\right)$, then (23) turns into

$$
\gamma\left(t_{a} ; \alpha v_{a}^{\mathrm{so}}, u_{a}, \lambda\right) \leq \max _{\varphi \in[0,1]}\left\{\varphi\left(1-\lambda \varphi^{p}\right)-(1-\varphi) k_{a}\right\}
$$

When $\varphi=\left(\left(1+k_{a}\right) / 5 \lambda\right)^{1 / 4}, \gamma$ attains its maximal bound $\frac{p}{p+1}\left(1+k_{a}\right)\left(\frac{1+k_{a}}{(p+1) \lambda}\right)^{1 / p}-k_{a}$.

(ii) $\alpha v_{a}^{\text {so }}+v_{a}^{*}<v_{a}^{\text {so }}$. In this case, considering also the increasing property of $t_{a}(v)$, and the fact that $v_{a}^{\mathrm{so}} /\left(\alpha v_{a}^{\mathrm{so}}+v_{a}^{*}\right) \leq v_{a}^{\mathrm{so}} / \alpha v_{a}^{\mathrm{so}}=1 / \alpha$, we get

$$
\begin{aligned}
& \gamma\left(t_{a} ; \alpha v_{a}^{\mathrm{so}}, u_{a}, \lambda\right) \leq \sup _{v_{a}^{\mathrm{a}}, v_{a}^{2} \geq 0} \frac{\left(t_{a}\left(\alpha v_{a}^{\mathrm{so}}+v_{a}^{*}\right)-\lambda t_{a}\left(\alpha v_{a}^{\mathrm{so}}+v_{a}^{*}\right)\right) v_{a}^{\mathrm{so}}-\left(\alpha v_{a}^{\mathrm{so}}+v_{a}^{*}-v_{a}^{\mathrm{so}}\right) u_{a}}{t_{a}\left(\alpha v_{a}^{\mathrm{so}}+v_{a}^{*}\right)\left(\alpha v_{a}^{\mathrm{sog}}+v_{a}^{*}\right)}
\end{aligned}
$$

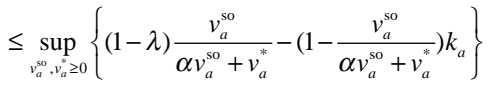

$$
\begin{aligned}
& \leq \frac{1}{\alpha}(1-\lambda)-\left(1-\frac{1}{\alpha}\right) k_{a}
\end{aligned}
$$

So summing up case (i) and (ii) completes the proof.
Based on the above analysis, we are going to seek the upper bounds on POA defined for this paper. Above all, we are firmly convinced that the following Lemma 3 holds.

Lemma 3. If the following Eq. (24) has a unique solution $\lambda_{p} \in[0,1]$

$\frac{1}{\alpha}(1-\lambda)-\left(1-\frac{1}{\alpha}\right) k_{a}=\frac{p}{p+1}\left(1+k_{a}\right)\left(\frac{1+k_{a}}{(p+1) \lambda}\right)^{1 / p}-k_{a}$

then $\lambda_{p}=z_{p}^{p} /(p+1)$, where $z_{p}$ is the unique solution larger than 1 to

$$
z^{p+1}-\left(1+k_{a}\right)(p+1) z+\alpha p\left(1+k_{a}\right)^{1+1 / p}=0
$$

In fact, just only need to set $\lambda_{p}=z_{p}^{p} /(p+1)$, and substitute it into the above equation with respect to $\lambda$, then rewriting the equation yields (25) that has one solution $z_{p}>1$.

By Lemma 2 and 3, we get

$$
\gamma\left(\mathcal{T}, \alpha v_{a}^{\mathrm{so}}, u, \lambda\right) \leq \frac{p\left(1+k_{a}\right)^{1+1 / p}}{p+1} z_{p}^{-1}-k_{a}
$$

$\gamma\left(\mathcal{T}, \alpha v_{a}^{\text {so }}, u, \lambda\right) \quad$ falls at interval $(0,1) \quad$ as $k_{a} \in\left\{k_{a} \in[0,2] \mid p\left(1+k_{a}\right)^{1 / p} /(p+1)<z_{p}\right\}$.

Now we set out to analyze an upper bound on $F\left(f, f^{*}\right)$ on the right hand side of the inequality (21).

Lemma 4. Let $\bar{c}_{0}$ be the average of optimal total system travel time with respect to network demand, and $\bar{\zeta}$ be the average of $\zeta_{w}, w \in W$ weighted by OD demand, where $\zeta_{w}$ solves $\left|\boldsymbol{R}_{w}\right|-1=\zeta_{w} \exp \left(\zeta_{w}+1\right)$, then it can be inferred that

$F\left(\boldsymbol{f}, \boldsymbol{f}^{*}\right) \quad \sum_{w \in W} \sum_{r \in R_{w}}\left(\frac{1-\mu}{\theta} \ln \left((1-\alpha) q^{w}\right)+\frac{\mu}{\theta} \ln f_{e r}^{w^{*}}-\frac{1}{\theta} \ln \alpha_{e r}^{w}\right)\left(f_{e r}^{w, s o}-f_{e r}^{\omega^{*}}-\bar{f}_{e r}^{w}\right)$

has the maximum value of $(1-\alpha) T^{\mathrm{so}} \mu K, K \quad \bar{\zeta} /\left(\theta \bar{c}_{0}\right)$.

Proof. Simple transformations of $F\left(f, f^{*}\right)$ results in

$$
\begin{aligned}
& F\left(\boldsymbol{f}, \boldsymbol{f}^{*}\right)=\sum_{w \in \boldsymbol{W}} \sum_{r \in \boldsymbol{R}_{w}}(1-\alpha) q^{w}\left(\frac{1}{\theta} \ln \left((1-\alpha) q^{w}\right)+\right. \\
& \left.\frac{\mu}{\theta} \ln \frac{f_{e r}^{w^{*}}}{(1-\alpha) q^{w}}-\frac{1}{\theta} \ln \alpha_{e r}^{w}\right)\left(\frac{f_{e r}^{w, s o}-\bar{f}_{e r}^{w}}{(1-\alpha) q^{w}}-\frac{f_{e r}^{w^{*}}}{(1-\alpha) q^{w}}\right)
\end{aligned}
$$

Setting $L_{r \max }=\max _{r \in \boldsymbol{R}_{w}} L_{r}$, and in view of the calculating formula (3) of allocating parameter, the above formula of $F\left(\boldsymbol{f}, \boldsymbol{f}^{*}\right)$ can be upper bounded as

$$
\begin{aligned}
& F\left(\boldsymbol{f}, \boldsymbol{f}^{*}\right) \leq \sum_{w \in W} \sum_{r \in \boldsymbol{R}_{w}}(1-\alpha) q^{w}\left(\frac{\mu}{\theta} \ln \frac{f_{e r}^{w^{*}}}{(1-\alpha) q^{w}}+\frac{1}{\theta} \ln \left((1-\alpha) q^{w}\right)-\right. \\
& \left.\frac{\gamma_{1}}{\theta} \ln \left(\frac{L_{e}}{\max _{r \in \boldsymbol{R}_{w}} L_{r}}\right)\right)\left(\frac{f_{e r}^{w, \mathrm{so}}-\bar{f}_{e r}^{w}}{(1-\alpha) q^{w}}-\frac{f_{e r}^{w^{*}}}{(1-\alpha) q^{w}}\right)
\end{aligned}
$$


Let $\quad a^{w}=\frac{1}{\mu} \ln \left((1-\alpha) q^{w}\right)-\frac{\gamma_{1}}{\mu} \ln \left(L_{e} / \max _{r \in \boldsymbol{R}_{w}} L_{r}\right)$, then (28) is simplified to be

$$
\begin{gathered}
F\left(\boldsymbol{f}, \boldsymbol{f}^{*}\right) \leq \sum_{w \in W}(1-\alpha) \frac{\mu}{\theta} q^{w} \sum_{r \in \boldsymbol{R}_{w}}\left(\ln \frac{f_{e r}^{w^{*}}}{(1-\alpha) q^{w}}+a^{w}\right) \\
\left(\frac{f_{e r}^{w, \text { so }}-\bar{f}_{e r}^{w}}{(1-\alpha) q^{w}}-\frac{f_{e r}^{w^{*}}}{(1-\alpha) q^{w}}\right) \\
\text { Let } y_{r}^{w}=\frac{f_{r}^{w, \text { so }}-\bar{f}_{r}^{w}}{(1-\alpha) q^{w}}, \quad x_{r}^{w}=\frac{f_{e r}^{w^{*}}}{(1-\alpha) q^{w}}, \text { then we should }
\end{gathered}
$$
only bound the right hand side of the following inequality

$$
F\left(\boldsymbol{f}, \boldsymbol{f}^{*}\right) \leq \sum_{w \in W}(1-\alpha) \frac{\mu}{\theta} q^{w} \sum_{r \in \boldsymbol{R}_{w}}\left(a^{w}+\ln x_{r}^{w}\right)\left(y_{r}^{w}-x_{r}^{w}\right)
$$

Keeping in mind that $\sum_{r \in \boldsymbol{R}_{w}} y_{r}^{w}=1$ and $\sum_{r \in \boldsymbol{R}_{w}} x_{r}^{w}=1$, the remaining work is to solve the following nonlinear programming problem.

$$
\max \mathrm{Z}_{2}(\boldsymbol{x}, \boldsymbol{y})=\sum_{r \in \boldsymbol{R}_{w}}\left(y_{r}-x_{r}\right)\left(a+\ln x_{r}\right)
$$

(MP2) s.t. $\quad \sum_{r \in \boldsymbol{R}_{w}} x_{r}=b$

$$
\sum_{r \in \boldsymbol{R}_{w}} y_{r}=b
$$$$
x_{r}, y_{r} \geq 0, r \in \boldsymbol{R}_{w}
$$

where $x_{r} \quad x_{r}^{w}, y_{r} \quad y_{r}^{w}, a \quad a^{w}, b \quad b^{w}=1$.

Let $\boldsymbol{x}$ be fixed, the objective function is linear in $\boldsymbol{y}$, so it is convenient to get $\boldsymbol{y}=(b, 0, \cdots, 0)^{T} \in{ }_{+}^{\left|\boldsymbol{R}_{w}\right| \times 1}$ as an optimality of $\boldsymbol{y}$, where $\left|\boldsymbol{R}_{w}\right|$ denotes the number of feasible paths between $w \in \boldsymbol{W}$. Thus the nonlinear programming problem can be rewritten as

$$
\begin{aligned}
\max & \mathrm{Z}_{2}(\boldsymbol{x})=\left(b-x_{1}\right)\left(a+\ln x_{1}\right)-\sum_{r \in \boldsymbol{R}_{w} \backslash\{1\}} x_{r}\left(a+\ln x_{r}\right) \\
(\mathrm{MP} 3) \text { s.t. } & \sum_{r \in \boldsymbol{R}_{w}} x_{r}=b \\
& x_{r} \geq 0, r \in \boldsymbol{R}_{w}
\end{aligned}
$$

Let $\lambda$ be the dual variable associated with the equation constraint, the Karush-Kuhn-Tucker (KKT) necessary conditions are

$\left(\frac{\partial \mathrm{Z}_{2}(\boldsymbol{x})}{\partial x_{r}}+\lambda\right) x_{r}=0, \frac{\partial \mathrm{Z}_{2}(\boldsymbol{x})}{\partial x_{r}}+\lambda \leq 0, x_{r} \geq 0, r \in \boldsymbol{R}_{w} \backslash\{1\}$

These conditions yield the following results

$$
\ln \frac{\left(\left|\boldsymbol{R}_{w}\right|-1\right) x_{1}}{b-x_{1}}=\frac{b}{x_{1}}, \quad \text { i.e. } \quad \frac{\left|\boldsymbol{R}_{w}\right|-1}{\zeta_{w}}=\exp \left(\zeta_{w}+1\right),
$$
$\zeta_{w}=\frac{b}{x_{1}}-1$

The corresponding objective value of (MP2) is $\mathrm{Z}_{2 \max }=b \zeta_{w}$, where $\zeta_{w}$ solves $\left|\boldsymbol{R}_{w}\right|-1=\zeta_{w} \exp \left(\zeta_{w}+1\right)$.
Thus, in the light of (29) and the optimal objective value of (MP2), we get

$$
F\left(f, f^{*}\right) \leq \sum_{w \in W}(1-\alpha) \frac{\mu}{\theta} q^{w} b \zeta_{w}=(1-\alpha) \frac{\mu}{\theta} \sum_{w \in W} q^{w} \zeta_{w}=(1-\alpha) \frac{\mu}{\theta} q \bar{\zeta},
$$

or $F\left(\boldsymbol{f}, \boldsymbol{f}^{*}\right) \leq(1-\alpha) \mu K T^{\mathrm{so}}$

where $\bar{\zeta}$ represents the average of $\zeta_{w}$ for every $w \in \boldsymbol{W}$, $K \bar{\zeta} /\left(\theta \bar{c}_{0}\right), \bar{c}_{0}=T^{\mathrm{so}} / q$.

Through the above analyses, the upper bounds on POA is attained in the following theorem.

Theorem 3. For link time function $t_{a} \in \mathcal{T}_{p}$, the price of anarchy induced by traveler behavior in Stackelberg stochastic user equilibrium with tax schemes is upper bounded as following

$$
\begin{aligned}
& \frac{T\left(\bar{v}+v^{*}\right)}{T^{\mathrm{so}}} \leq \frac{z_{p}^{p} /(p+1)}{1-\left(\frac{p\left(1+k_{a}\right)^{1+1 / p}}{p+1} z_{p}^{-1}-k_{a}\right)}+ \\
& \cdot \frac{1}{\left(1-\left(\frac{p\left(1+k_{a}\right)^{1+1 / p}}{p+1} z_{p}^{-1}-k_{a}\right)\right) T^{\mathrm{so}}}(1-\alpha) \mu K T^{\mathrm{so}} \\
& =\frac{\left(1+k_{a}\right)(p+1) z_{p}-\alpha p\left(1+k_{a}\right)^{1+1 / p}}{(p+1)\left(1+k_{a}\right) z_{p}-p\left(1+k_{a}\right)^{1+1 / p}}+
\end{aligned}
$$

$\frac{(1-\alpha)(p+1) \mu K z_{p}}{(p+1)\left(1+k_{a}\right) z_{p}-p\left(1+k_{a}\right)^{1+1 / p}}$

where

$$
\begin{aligned}
& k_{a} \in\left\{k_{a} \in[0,2] \mid \frac{p\left(1+k_{a}\right)^{1 / p}}{p+1}<z_{p}\right\} \cap\left\{k_{a} \in[0,2] \mid 1<z_{p}=\right. \\
& \left.z_{p}\left(\alpha, k_{a}\right) \leq(p+1)^{1 / p}, z_{p} \text { solves equation (25) }\right\}
\end{aligned}
$$

Proof. Let $\lambda_{p}=z_{p}^{p} /(p+1)$, where $z_{p}$ is the unique solution larger than one to (25). Then, substituting $\lambda_{p}$, and inequality (33), the upper bound of $F\left(\boldsymbol{f}, \boldsymbol{f}^{*}\right)$ into (22), we obtain formula (34).

It is convenient to get $z_{p} \leq(p+1)^{1 / p}$ by $\lambda_{p}=z_{p}^{p} /(p+1) \in[0,1], \quad$ where $z_{p}=z_{p}\left(\alpha, k_{a}\right)>1$ solves equation (25). Connecting with value range of $k_{a}$ that ensures $\gamma\left(\mathcal{T}, \alpha v_{a}^{\text {so }}, u, \lambda\right) \in(0,1)$, we obtain (35).

The POA upper bound, for BPR link time function, in Stackelberg stochastic network assignment using SCALE policy, is the right hand side of (34). It is worth pointing out that, since $z_{p}>1$, inequality (26) turns into $\gamma\left(\mathcal{T}, \alpha v_{a}^{\text {so }}, u, \lambda\right) \leq p\left(1+k_{a}\right)^{1+1 / p} /(p+1)-k_{a}$, which can be 
used to replace (26) to calculate the upper bound but generating even less tight bound.

Corollary 1. When $\mu=1$ the POA upper bound expressed by (34) changes to be

$$
\begin{aligned}
& \frac{T\left(\bar{v}+v^{*}\right)}{T^{\mathrm{so}}}=\frac{\left(1+k_{a}\right)(p+1) z_{p}-\alpha p\left(1+k_{a}\right)^{1+1 / p}}{(p+1)\left(1+k_{a}\right) z_{p}-p\left(1+k_{a}\right)^{1+1 / p}}+ \\
& -\frac{(1-\alpha)(p+1) K z_{p}}{(p+1)\left(1+k_{a}\right) z_{p}-d\left(1+k_{a}\right)^{1+1 / p}}
\end{aligned}
$$

which coincides with that obtained by MNL model that is induced from the CNL model (2) if $\mu=1$.

\section{POA UPPER BOUND FOR LOW-DEGREE LINK TIME FUNCTIONS}

Now, we are going to display graphically the upper bounds for SUE. The analytical solution to the equation (25) of high-degree (especially $p \geq 5$ ) is difficult to obtain, so we take the cases of low degree of power law for examples. In the remaining space, numerical analyses with figures are presented to examine the relationship of POA upper bound with weak Stackelberg controlling factor $\alpha$ and tax coefficient $k_{a}$.

Furthermore, to verify the correctness of the foregoing conclusions, and to achieve better understanding of the issue studied, we consider the POA upper bounds for affine link time function, a special case of the BPR function, in Stackelberg network under SUE and tax scheme.

According to the analysis process for BPR stated above, we have $\lambda=\left(1+k_{a}\right)(1+\sqrt{1-\alpha}) / 2$, which at the same time implies $\quad k_{a} \leq 2 /(1+\sqrt{1-\alpha})-1=(1-\sqrt{1-\alpha}) /(1+\sqrt{1-\alpha})$ owing to $\lambda \in[0,1]$. Then, (18) and (19) generate

$$
\gamma\left(\mathcal{T}, \alpha v_{a}^{\mathrm{so}}, u, \lambda\right) \leq \frac{\left(1+k_{a}\right)}{2(1+\sqrt{1-\alpha})}-k_{a}
$$

satisfying $0<\gamma\left(\mathcal{T}, \alpha v_{a}^{\text {so }}, u, \lambda\right)<1$ for all $k_{a} \in[0,2]$.

Thus using similar analyses in theorem 3 , we get the following corollary.

Corollary 2. For affine link time functions, the upper bound of POA with the SCALE strategy is as following:

$$
\begin{aligned}
& \frac{T\left(\overline{\boldsymbol{v}}+\boldsymbol{v}^{*}\right)}{T^{\mathrm{so}}} \leq \frac{\left(1+k_{a}\right)(1+\sqrt{1-\alpha})^{2}}{2(1+\sqrt{1-\alpha})-1+(2 \sqrt{1-\alpha}+1) k_{a}}+ \\
& \frac{2(1-\alpha)(1+\sqrt{1-\alpha}) \mu K}{2(1+\sqrt{1-\alpha})-1+(2 \sqrt{1-\alpha}+1) k_{a}}
\end{aligned}
$$

where $0 \leq k_{a} \leq(1-\sqrt{1-\alpha}) /(1+\sqrt{1-\alpha})$.
Formula (37) indicates that the upper bound of POA in the stochastic circumstances is not increasing with respect to $k_{a}$, which is also explicit from the non-positive derivative of the right hand side of $(37),-\Lambda^{2}(1+\Lambda / 2)(1+\Lambda) /$ $\left(2(1+\Lambda)^{2}\left(1+k_{a}\right)^{2}\right), \Lambda=2 \sqrt{1-\alpha}$. It is also clear that the relation of POA with nested parameter $\mu$ is strictly nondecreasing.

$\bar{\zeta}$-value affects $K$-value little from ref. [16]. If the values $\bar{\zeta}$ and $\bar{c}_{0}$ are fixed, $K$ is determined by and decreasing with parameter $\theta$, that is to say, a weaker randomness means a smaller $K$. For $K=0.1$, the relation of POA with Stackelberg controlling fraction $\alpha$ and nested degree $\mu$ is depicted as Fig. (1).

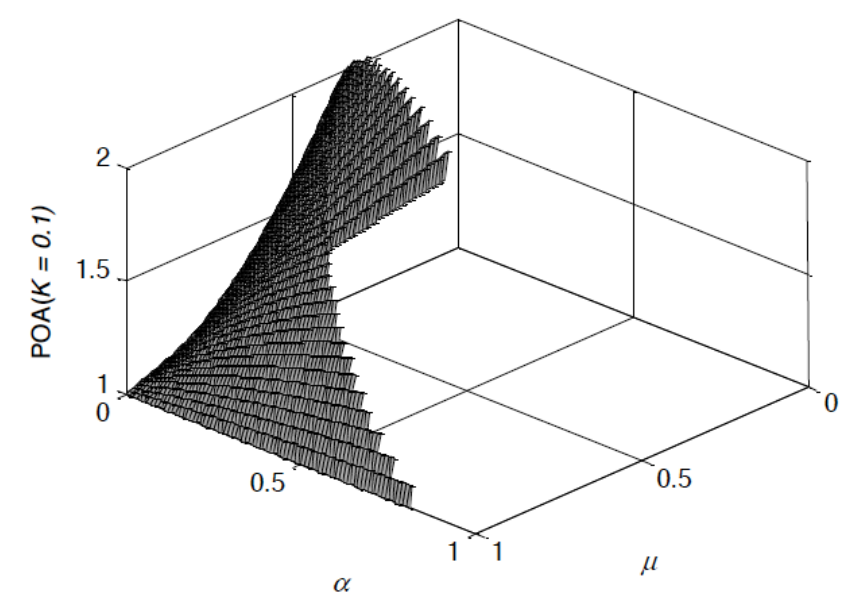

Fig. (1). Upper bound of POA of Stackelberg network under SUE.

Corollary 3. The price of anarchy induced by traveler behavior in Stackelberg stochastic user equilibrium with tax schemes is upper bounded for typical BPR link time function $t_{a}\left(v_{a}\right)=t_{a}^{0}\left(1+0.15\left(v_{a} / C_{a}\right)^{4}\right)$, as following

$$
\frac{T\left(\overline{\boldsymbol{v}}+\boldsymbol{v}^{*}\right)}{T^{\mathrm{so}}} \leq \frac{5\left(1+k_{a}\right) z_{4}-4 \alpha\left(1+k_{a}\right)^{5 / 4}}{5\left(1+k_{a}\right) z_{4}-4\left(1+k_{a}\right)^{5 / 4}}+\frac{5(1-\alpha) \mu K z_{4}}{5\left(1+k_{a}\right) z_{4}-4\left(1+k_{a}\right)^{5 / 4}}
$$

where $t_{a}^{0}$ and $C_{a}$ are respectively the link free-flow travel time and link capacity.

The upper bounds are not increasing with tax coefficient $k_{a}$, and that if $\alpha=1$ meaning complete controlling of travelers by the network manager, the Stackelberg network system achieves optimum without loss of efficiency. For different values of $K$, the above process is carried out, such as for $K=0.1$ and $K=0.2$, the POA $-k_{a}$ relationship shows that: for the same value of $\alpha$, a bigger $K$ means a bigger the upper bound of POA and a slower decreasing amplitude of inefficiency upper bound. Corollary3 also implies that, when $\alpha$ takes a larger value, the decrease rate of upper bound arrives a reduced magnitude, i.e., a diminishing marginal effect of the central controlling fraction $\alpha$ to decrease upper bound. 


\section{CONCLUSION}

Under the assumptions of separable nondecreasing convex link travel time, fixed OD demand, and additional tax scheme, this paper investigates the POA upper bounds on stochastic selfish routing, accompanied by the centrally controlled Stackelberg flow, jointly leading to SUE. Intensive analyses come to some conclusions. For general link time functions, we establish the equivalent variational inequality formulation for the CNL-SUE, induced by weak Stackelberg strategy as well as a tax scheme, with further presentation of inefficiency upper bounds. Commonly used BPR function and its affine form are focused on and shown that a larger value of the central controlling fraction $\alpha$ leads to a diminishing efficiency loss and a diminishing marginal effect of $\alpha$. If travelers are completely controlled, the Stackelberg transport network system achieves full efficiency. For the same value of $\alpha$, the smaller the value of $K$ (or weaker randomness if the other two factor of $\bar{\zeta}$ and $\bar{c}_{0}$ are fixed), the smaller the POA upper bound and the slower its decreasing amplitude. In the circumstance of SUE for typical or affine BPR functions, network taxes can reduce to some degree the performance degradation due to selfish behavior.

\section{CONFLICT OF INTEREST}

The authors confirm that this article content has no conflict of interest.

\section{ACKNOWLEDGEMENTS}

This research was supported by the National Natural Science Foundation of China (Grant No. 51238008 and No. 51468020), and the Natural Science Foundation of Jiangxi Province (Grant No. 20142BAB207016).

\section{REFERENCES}

[1] E. Koutsoupias, and C. Papadimitriou, "Worst-case equilibria," In: Proceedings of the 16th Annual Conference on Theoretical Aspects of Computer Science, Springer-Verlag, pp. 404-413, 1999.

[2] C. H. Papadimitriou," "Algorithms, games, and the internet," In: Proceedings of the 33rd Annual ACM Symposium on the Theory of Computing, ACM, Hersonissos, Crete, Greece, pp. 749-753, 2001.

[3] T. Roughgarden, and É. Tardos, "How bad is selfish routing," Journal of the ACM (JACM), vol. 49, no. 2, pp. 236-259, 2002.

[4] J. R. Correa, A. S. Schulz, N. E. Stier-Moses, "On the inefficiency of equilibria in congestion games," In: Jünger, M., Kaibel, V. (Eds.), Proceedings of the 11th International Integer Programming and Combinatorial Optimization Conference (IPCO). Berlin, Germany. In: Lecture Notes in Computer Science, vol. 3509. Springer, Heidelberg, pp. 167-181, 2005.

[5] H. Yang, W. Xu, and B. Heydecker, "Bounding the efficiency of road pricing," Transportation Research Part E: Logistics and Transportation Review, vol. 46, no. 1, pp. 90-108, 2010.
[6] C. K. Chau, and K. M. Sim, "The price of anarchy for non-atomic congestion games with symmetric cost maps and elastic demands," Operations Research Letters, vol. 31, no. 5, pp. 327-334, 2003.

[7] V. Bonifaci, T. Harks, and G. Schäfer, "Stackelberg routing in arbitrary networks, " Mathematics Of Operations Research, vol. 35, no. 2, pp. 330-346, 2010.

[8] T. Harks, "Stackelberg strategies and collusion in network games with splittable flow," Theory Of Computing Systems, vol. 48, no. 4, pp. 781-802, 2011.

[9] Y.A. Korilis, A. A. Lazar, and A. Orda, "Achieving network optima using Stackelberg routing strategies," IEEE/ACM Transactions on Networking (TON), vol. 5, no. 1, pp. 161-173, 1997.

[10] G. Karakostas, and S. G. Kolliopoulos, "Stackelberg strategies for selfish routing in general multicommodity networks," Algorithmica, vol. 53, no. 1, pp. 132-153, 2009.

[11] T. Roughgarden, "Stackelberg scheduling strategies," SIAM Journal on Computing, 2004, vol. 33, no. 2, pp. 332-350. Conference version in STOC, pp. 104-113, 2001.

[12] N. E. S. Moses, "Selfish Versus Coordinated Routing in Network Games," Doctorial thesis of Massachusetts Institute of Technology, Sloan School of Management, Operations Research Center, 2004.

[13] A. C. Pigou, "Wealth and Welfare," Macmillan, London, 1920.

[14] C. Swamy, "The effectiveness of Stackelberg strategies and tolls for network congestion games," In: Proceedings of the 18th ACMSIAM Symposium on Discrete Algorithms, SIAM, Philadelphia, pp. 1133-1142, 2007.

[15] T. Domencich, and D. M. Fadden, Urban Travel Demand - A Behavioural Analysis," Amsterdam: North Holland, 1975.

[16] X. Guo, H. Yang, and T. L. Liu, "Bounding the inefficiency of logit-based stochastic user equilibrium," European Journal of Operational Research, vol. 201, no. 2, pp. 463-469, 2010.

[17] X. Yu, H. Huang, and T. Liu, "Efficiency Loss of the Multiclass Stochastic Traffic Equilibrium Assignment with Fixed Demand," Journal of Transportation Systems Engineering and Information Technology, vol. 9, no. 4, pp. 83-89, 2009.

[18] J. N. Prashker, and S. Bekhor, "Investigation of stochastic network loading procedures. Transportation Research Record," Journal of the Transportation Research Board, vol. 1645, no. 1, pp. 94-102, 1998.

[19] M. A. Bierlaire, "Theoretical analysis of the cross-nested logit model," Annals Of Operations Research, vol. 144, no. 1, pp. 287300, 2006.

[20] P. Vovsha, "Application of cross-nested logit model to mode choice in Tel Aviv, Israel, metropolitan area. Transportation Research Record," Journal of the Transportation Research Board, vol. 1607 , no. 1, pp. 6-15, 1997.

[21] T. Roughgarden, "Selfish routing and the price of anarchy. Cambridge," Massachusetts: The MIT Press, 2005.

[22] J. Correa, and N. Stier-Moses, "Stackelberg routing in atomic network games," Columbia Working Paper No. DRO-2007-03, 2007: http://papers.ssrn.com/sol3/papers.cfm?abstract_id=987115.

[23] M. J. Smith, "The existence, uniqueness and stability of traffic equilibrium," Transportation Research B: Methodological, vol. 13, no. 4, pp. 295-304, 1979.

[24] S. Bekhor, M. E. B. Akiva, and M. S. Ramming, "Evaluation of choice set generation algorithms for route choice models," Annals Of Operations Research, vol. 144, no. 1, pp. 235-247, 2006.

[25] T. Roughgarden, and É. Tardos, "Bounding the inefficiency of equilibria in nonatomic congestion games," Games and Economic Behavior, vol. 47, no. 2, pp. 389-403, 2004.

This is an open access article licensed under the terms of the Creative Commons Attribution Non-Commercial License (http://creativecommons.org/licenses/by-nc/3.0/) which permits unrestricted, non-commercial use, distribution and reproduction in any medium, provided the work is properly cited. 